\title{
Hilfer-Hadamard Nonlocal Integro-Multipoint Fractional Boundary Value Problems
}

\author{
Chanon Promsakon, ${ }^{1}$ Sotiris K. Ntouyas $\mathbb{D}^{2,3}$ and Jessada Tariboon $\mathbb{D}^{1}$ \\ ${ }^{1}$ Intelligent and Nonlinear Dynamic Innovations Research Center, Department of Mathematics, Faculty of Applied Science, \\ King Mongkut's University of Technology North Bangkok, Bangkok 10800, Thailand \\ ${ }^{2}$ Department of Mathematics, University of Ioannina, 45110 Ioannina, Greece \\ ${ }^{3}$ Nonlinear Analysis and Applied Mathematics (NAAM)-Research Group, Department of Mathematics, Faculty of Science, \\ King Abdulaziz University, P.O. Box 80203, Jeddah 21589, Saudi Arabia
}

Correspondence should be addressed to Jessada Tariboon; jessada.t@sci.kmutnb.ac.th

Received 24 June 2021; Accepted 1 December 2021; Published 17 December 2021

Academic Editor: Feliz Minhós

Copyright (C) 2021 Chanon Promsakon et al. This is an open access article distributed under the Creative Commons Attribution License, which permits unrestricted use, distribution, and reproduction in any medium, provided the original work is properly cited.

\begin{abstract}
This paper is concerned with the existence and uniqueness of solutions for a new class of boundary value problems, consisting by Hilfer-Hadamard fractional differential equations, supplemented with nonlocal integro-multipoint boundary conditions. The existence of a unique solution is obtained via Banach contraction mapping principle, while the existence results are established by applying Schaefer and Krasnoselskii fixed point theorems as well as Leray-Schauder nonlinear alternative. Examples illustrating the main results are also constructed.
\end{abstract}

\section{Introduction}

The fractional calculus has always been an interesting research topic for many years. This is because fractional differential equations describe many real-world process related to memory and hereditary properties of various materials more accurately as compared to classical-order differential equations. Fractional differential equations arise in lots of engineering and clinical disciplines which include biology, physics, chemistry, economics, signal and image processing, and control theory (see the monographs and papers in [1-8]).

Various types of fractional derivatives were introduced among which the following Riemann-Liouville and Caputo derivatives are the most widely used ones.

(1) Riemann-Liouville derivative. For $n-1<\alpha<n$, the derivative of $u$ is

$$
{ }^{\mathrm{RL}} D_{a}^{\alpha} u(t):=D^{n} I_{a}^{n-\alpha} u(t)=\frac{1}{\Gamma(n-\alpha)}\left(\frac{d}{d t}\right)^{n} \int_{a}^{t}(t-s)^{n-\alpha-1} u(s) d s
$$

(2) Caputo derivative. For $n-1<\alpha<n$, the derivative of $u$ is

$$
{ }^{C} D_{a}^{\alpha} u(t):=I_{a}^{n-\alpha} D^{n} u(t)=\frac{1}{\Gamma(n-\alpha)} \int_{a}^{t}(t-s)^{n-\alpha-1}\left(\frac{d}{d s}\right)^{n} u(s) d s,
$$

where $D^{n}=(d / d t)^{n}$. Both Riemann-Liouville and Caputo derivatives are defined via fractional integral, the 
Riemann-Liouville fractional integral, which is defined by

$$
I_{a}^{\alpha} u(t)=\frac{1}{\Gamma(\alpha)} \int_{a}^{t}(t-s)^{\alpha-1} u(s) d s, \quad n-1<\alpha<n
$$

A generalization of derivatives of both RiemannLiouville and Caputo was given by R. Hilfer in [9], known as the Hilfer fractional derivative of order $\alpha$ and a type $\beta$ $\epsilon[0,1]$, which interpolates between the RiemannLiouville and Caputo derivatives, since it is reduced to the Riemann-Liouville and Caputo fractional derivatives when $\beta=0$ and $\beta=1$, respectively. The Hilfer fractional derivative of order $\alpha$ and parameter $\beta$ of a function $u$ is defined by

$$
{ }^{H} D_{a}^{\alpha, \beta} u(t)=I_{a}^{\beta(n-\alpha)} D^{n} I_{a}^{(1-\beta)(n-\alpha)} u(t),
$$

where $n-1<\alpha<n, 0 \leq \beta \leq 1, t>a, D=d / d t$. Some properties and applications of the Hilfer derivative are given in $[10,11]$ and references cited therein. Initial value problems involving Hilfer fractional derivatives were studied by several authors (see, for example, [12-15] and references therein). Nonlocal boundary value problems for Hilfer fractional derivative were studied in $[16,17]$.

The Hadamard fractional calculus contains fractional derivative and integral with respect to the logarithmic function while someone say that it is generalized of the derivative $(t(d / d t))^{\alpha}$, where $\alpha$ is an arbitrary order. The Hadamard calculus can be obtained by replacing as $d / d t$ $\longrightarrow t d / d t,(t-s)^{(\cdot)} \longrightarrow\left(\log _{e} t-\log _{e} s\right)^{(\cdot)}$, and $d s \longrightarrow(1 / s) d$ $s$ in (1)-(3). In the same way, the concept of HilferHadamard derivative is arrived by the modified definition in (4). Existence and uniqueness of solutions for system of Hilfer-Hadamard sequential fractional differential equations with two point boundary conditions were studied in [18].

In this paper, we study existence and uniqueness of solutions for boundary value problems for Hilfer-Hadamard fractional differential equations with nonlocal integromultipoint boundary conditions,

$$
\begin{cases}{ }^{\mathrm{HH}} D_{1}^{\alpha, \beta} x(t)=f(t, x(t)), & t \in[1, T] \\ x(1)=0, & \sum_{i=1}^{m} \theta_{i} x\left(\xi_{i}\right)=\lambda^{H} I_{1}^{\delta} x(\eta)\end{cases}
$$

where ${ }^{\mathrm{HH}} D_{1}^{\alpha, \beta}$ is the Hilfer-Hadamard fractional derivative of order $\alpha \in(1,2]$ and type $\beta \in[0,1], \theta_{i}, \lambda \in \mathbb{R}, i=1,2, \cdots$, $m$, are given constants, and $f:[1, T] \times \mathbb{R} \longrightarrow \mathbb{R}$ is a given continuous function, ${ }^{H} I^{\delta}$ is the Hadamard fractional integral of order $\delta>0$, and $\eta, \xi_{i} \in(1, T), i=1,2, \cdots, m$, are given points.

Existence and uniqueness results are established by using classical fixed point theorems. We make use of Banach's fixed point theorem to obtain the uniqueness result, while Schaefer and Krasnoselskii's fixed point theorem [19] as well as nonlinear alternative of Leray-Schauder type [20] is applied to obtain the existence results for the problem (5).

The paper is constructed as follows: in Section 2, we recall some basic facts needed in our study. The main results are proved in Section 3. Examples illustrating the main results are presented in Section 4.

\section{Preliminaries}

In this section, some basic definitions, lemmas, and theorems are mentioned.

Definition 1 (Hadamard fractional integral [2]). The Hadamard fractional integral of order $\alpha>0$ for a function $f:[a$, $\infty) \longrightarrow \mathbb{R}$ is defined as

$$
{ }^{H} I_{a}^{\alpha} f(t)=\frac{1}{\Gamma(\alpha)} \int_{a}^{t}\left(\log \frac{t}{\tau}\right)^{\alpha-1} \frac{f(\tau)}{\tau} d \tau, \quad t>a,
$$

provided the integral exists, where $\log ()=.\log _{e}($.$) .$

Definition 2 (Hadamard fractional derivative [2]). The Hadamard fractional derivative of order $\alpha>0$, applied to the fuction $f:[a, \infty) \longrightarrow \mathbb{R}$, is defined as follows:

$$
{ }_{H} D_{a}^{\alpha} f(t)=\delta^{n}\left({ }^{H} I_{a}^{n-\alpha} f\right)(t), n=[\alpha]+1,
$$

where $\delta^{n}=(t(d / d t))^{n}$ and $[\alpha]$ denotes the integer part of the real number $\alpha$.

Definition 3 (Hilfer-Hadamard fractional derivative [11]). Let $n-1<\alpha<n$ and $0 \leq \beta \leq 1, f \in L^{1}(a, b)$. The HilferHadamard fractional derivative of order $\alpha$ and tybe $\beta$ of $f$ is defined as

$$
\begin{aligned}
\left({ }^{\mathrm{HH}} D_{a}^{\alpha, \beta} f\right)(t) & =\left({ }^{H} I_{a}^{\beta(n-\alpha)} \delta^{n H} I_{a}^{(n-\alpha)(1-\beta)} f\right)(t) \\
& =\left({ }^{H} I_{a}^{\beta(n-\alpha)} \delta^{n H} I_{a}^{(n-\gamma)} f\right)(t) \\
& =\left({ }^{H} I_{a}^{\beta(n-\alpha)}{ }_{H} D_{a}^{\gamma} f\right)(t), \quad \gamma=\alpha+n \beta-\alpha \beta,
\end{aligned}
$$

where ${ }^{H} I_{a}^{(.)}$and ${ }_{H} D_{a}^{(.)}$is the Hadamard fractional integral and derivative defined by (6) and (7), respectively.

The Hilfer-Hadamard fractional derivative may be viewed as interpolating between Hadamard and CaputoHadamard fractional derivatives. Indeed, for $\beta=0$, this derivative reduces to the Hadamard fractional derivative while for $\beta=1$, it leads the Caputo-Hadamard derivative defined by

$$
{ }_{H}^{C} D_{a}^{\alpha} f(t)=\left({ }^{H} I_{a}^{n-\alpha} \delta^{n} f\right)(t), \quad n=[\alpha]+1 .
$$

We recall the following known theorem by Kilbas et al. [2] which will be used in the following. 
Theorem 4 ([2]). Let $\alpha>0,0 \leq \beta \leq 1, \gamma=\alpha+n \beta-\alpha \beta, n=[$ $\alpha]+1$, and $0<a<b<\infty$. If $f \in L^{1}(a, b)$ and $\left({ }^{H} I_{a}^{n-\gamma} f\right)(t) \in$ $A C_{\delta}^{n}[a, b]$, then

$$
\begin{aligned}
& { }^{H} I_{a}^{\alpha}\left({ }^{H H} D_{a}^{\alpha, \beta} f\right)(t)={ }^{H} I_{a}^{\gamma}\left({ }^{H H} D_{a}^{\gamma} f\right)(t) \\
& \quad=f(t)-\sum_{j=0}^{n-1} \frac{\left(\delta^{(n-j-1)}\left({ }^{H} I_{a}^{n-\gamma} f\right)\right)(a)}{\Gamma(\gamma-j)}\left(\log \frac{t}{a}\right)^{\gamma-j-1} .
\end{aligned}
$$

Since $\gamma \in[\alpha, n]$, then the $\Gamma(\gamma-j)$ exists for all $j=1,2, \cdots$ , $n-1$.

Finally, we will use the following well-known fixed point theorems on Banach space for proving the existence and uniqueness of the solutions to Hilfer-Hadamard fractional boundary value problem (5).

Theorem 5 (Banach's contraction principle [21]). Let $X$ be a Banach space, $D \subset X$ be closed, and $T: D \longrightarrow D$ be a contraction (i.e., there exists a constant $L \in(0,1)$ such that for any $x, y \in X,|T x-T y| \leq L|x-y|)$. Then, $T$ has a unique fixed point on $X$.

Theorem 6 (Krasnoselskii's fixed point theorem)[19]. Let $Y$ be a bounded, closed, convex, and nonempty subset of a Banach space X. Let $F_{1}$ and $F_{2}$ be the operators satisfying the following conditions: (i) $F_{1} y_{1}+F_{2} y_{2} \in Y$ whenever $y_{1}, y_{2} \in Y$; (ii) $F_{1}$ is compact and continuous; and (iii) $F_{2}$ is a contraction mapping. Then, there exists $y \in Y$ such that $y=F_{1} y+F_{2} y$.

Theorem 7 (Schaefer fixed point theorem [22]). Let $T: E$ $\longrightarrow$ E be a completely continuous operator (i.e., a continuous map $T$ restricted to any bounded set in $E$ is compact). Let $\varepsilon$ $(T)=\{x \in E: x=\lambda T(x), 0 \leq \lambda \leq 1\}$. Then, either the set $\varepsilon(T$ ) is unbounded or $T$ has at least one fixed point.
Theorem 8 (Nonlinear alternative for single-valued maps) ([20]). Let E be a Banach space, $C$ a closed, convex subset of $E, U$ an open subset of $C$, and $0 \in U$. Suppose that $\mathscr{A}: \bar{U}$ $\longrightarrow C$ is continuous and compact (that is, $\mathscr{A}(\bar{U})$ is a relatively compact subset of C) map. Then, either

(i) $\mathscr{A}$ has a fixed point in $\bar{U}$, or

(ii) there is ax $\in \partial U$ (the boundary of $U$ in $C$ ) and $\lambda \in($ $0,1)$ with $x=\lambda \mathscr{A}(x)$

\section{Main Results}

In this section, we prove existence and uniqueness of solutions for nonlinear Hilfer-Hadamard fractional boundary value problem (5). Firstly, we start by proving a basic lemma concerning a linear variant of the boundary value problem (5), which will be used to transform the boundary value problem (5) into an equivalent integral equation. In this case, $n=[\alpha]+1=2$; then, we have $\gamma=\alpha+(2-\alpha) \beta$.

Lemma 9. Let $h \in C([1, T], \mathbb{R})$ and $\Lambda \neq 0$, where

$$
\Lambda=\sum_{i=1}^{m} \theta_{i}\left(\log \xi_{i}\right)^{\gamma-1}-\lambda \frac{\Gamma(\gamma)}{\Gamma(\gamma+\delta)}(\log \eta)^{\gamma+\delta-1}
$$

Then, $x$ is a solution of the following linear HilferHadamard fractional differential equation:

$$
{ }^{H H} D_{1}^{\alpha, \beta} x(t)=h(t), 1<\alpha \leq 2, \quad t \in[1, T],
$$

supplemented with the boundary conditions in (5), if and only if

$$
\begin{aligned}
x(t)= & { }^{H} I_{1}^{\alpha} h(t)+\frac{(\log t)^{\gamma-1}}{\Lambda}\left\{\lambda\left({ }^{H} I_{1}^{\alpha+\delta} h\right)(\eta)-\sum_{i=1}^{m} \theta_{i}\left({ }^{H} I_{1}^{\alpha} h\right)\left(\xi_{i}\right)\right\}=\frac{1}{\Gamma(\alpha)} \int_{1}^{t}\left(\log \frac{t}{\tau}\right)^{\alpha-1} \frac{h(\tau)}{\tau} d \tau \\
& +\frac{(\log t)^{\gamma-1}}{\Lambda}\left\{\lambda \frac{1}{\Gamma(\alpha+\delta)} \int_{1}^{\eta}\left(\log \frac{\eta}{\tau}\right)^{\alpha+\delta-1} \frac{h(\tau)}{\tau} d \tau-\sum_{i=1}^{m} \theta_{i} \frac{1}{\Gamma(\alpha)} \int_{1}^{\xi_{i}}\left(\log \frac{\xi_{i}}{\tau}\right)^{\alpha-1} \frac{h(\tau)}{\tau} d \tau\right\}, \quad t \in[1, T] .
\end{aligned}
$$

Proof. By taking the Hadamard fractional integral of order $\alpha$ from 1 to $t$ on both sides of (12) and using Theorem 4, it follows that

$$
x(t)-\sum_{j=0}^{1} \frac{\left(\delta^{(2-j-1)}\left({ }_{H} I_{1^{+}}^{2-\gamma} x\right)\right)(1)}{\Gamma(\gamma-j)}(\log t)^{\gamma-j-1}={ }^{H} I_{1}^{\alpha} h(t) .
$$

Then, we have

$$
\begin{aligned}
x(t) & -\frac{\delta\left({ }_{H} I_{1^{+}}^{2-\gamma} x\right)(1)}{\Gamma(\gamma)}(\log t)^{\gamma-1} \\
& -\frac{\left({ }_{H} I_{1^{+}}^{2-\gamma} x\right)(1)}{\Gamma(\gamma-1)}(\log t)^{\gamma-2}={ }^{H} I_{1}^{\alpha} h(t) .
\end{aligned}
$$


Equation (15) can be rewritten by

$$
x(t)=c_{0}(\log t)^{\gamma-1}+c_{1}(\log t)^{\gamma-2}+\frac{1}{\Gamma(\alpha)} \int_{1}^{t} \frac{h(s)}{s}\left(\log \frac{t}{s}\right)^{\alpha-1} d s,
$$

where $c_{0}, c_{1}$ are arbitrary constants. Now, the first boundary condition $x(1)=0$ together with (16) yields $c_{1}=0$, since $\gamma$ $\in[\alpha, 2]$. Putting $c_{1}=0$ in (16), we get

$$
x(t)=c_{0}(\log t)^{\gamma-1}+\frac{1}{\Gamma(\alpha)} \int_{1}^{t}\left(\log \frac{t}{s}\right)^{\alpha-1} \frac{h(s)}{s} d s .
$$

Next, the second boundary condition $\sum_{i=1}^{m} \theta_{i} x\left(\xi_{i}\right)=\lambda^{H}$ $I_{1}^{\delta} x(\eta)$ together with (17) yields

$$
c_{0}=\frac{1}{\Lambda}\left\{\lambda^{H} I_{1}^{\alpha+\delta} h(\eta)-\sum_{i=1}^{m} \theta_{i}^{H} I_{1}^{\alpha} h\left(\xi_{i}\right)\right\} .
$$

Substituting the value of $c_{0}$ in (17), we get equation (13) as desired.

The converse follows by direct computation. The proof is completed.

Let us introduce the Banach space $X=C([1, T], \mathbb{R})$ endowed with the norm defined by $\|x\|:=\max _{t \in[1, T]}|x(t)|$.

In view of Lemma 9, we define an operator $\mathscr{F}: X \longrightarrow X$, where

$$
\begin{aligned}
(\mathscr{F} x)(t)= & \frac{1}{\Gamma(\alpha)} \int_{1}^{t}\left(\log \frac{t}{\tau}\right)^{\alpha-1} \frac{f(\tau, x(\tau))}{\tau} d \tau \\
& +\frac{(\log t)^{\gamma-1}}{\Lambda}\left\{\frac{\lambda}{\Gamma(\alpha+\delta)} \int_{1}^{\eta}\left(\log \frac{\eta}{\tau}\right)^{\alpha+\delta-1} \frac{f(\tau, x(\tau))}{\tau} d \tau\right. \\
& \left.-\sum_{i=1}^{m} \theta_{i} \frac{1}{\Gamma(\alpha)} \int_{1}^{\xi_{i}}\left(\log \frac{\xi_{i}}{\tau}\right)^{\alpha-1} \frac{f(\tau, x(\tau))}{\tau} d \tau\right\}, \quad t \in[1, T] .
\end{aligned}
$$

In the following, for convenience, we put

$\Omega=\frac{(\log T)^{\alpha}}{\Gamma(\alpha+1)}+\frac{(\log T)^{\gamma-1}}{|\Lambda|}\left\{\frac{|\lambda|(\log \eta)^{\alpha+\delta}}{\Gamma(\alpha+\delta+1)}+\sum_{i=1}^{m}\left|\theta_{i}\right| \frac{\left(\log \xi_{i}\right)^{\alpha}}{\Gamma(\alpha+1)}\right\}$.

We need the following hypotheses in the sequel:

$\left(H_{1}\right)$. There exists a constant $l>0$ such that for all $t \in[1, T]$ and $u_{i} \in \mathbb{R}, i=1,2$,

$$
\left|f\left(t, u_{1}\right)-f\left(t, u_{2}\right)\right| \leq l\left|u_{1}-u_{2}\right| .
$$

$\left(\mathrm{H}_{2}\right)$. There exists a continuous nonnegative function $\phi \in C$ $\left([1, T], \mathbb{R}^{+}\right)$such that

$$
|f(t, u)| \leq \phi(t), \quad \text { for each }(t, u) \in[1, T] \times \mathbb{R} \text {. }
$$

$\left(H_{3}\right)$. There exists a real constant $M>0$ such that for all $t$ $\in[1, T], u \in \mathbb{R}$,

$$
|f(t, u)| \leq M
$$

$\left(H_{4}\right)$. There exist $p \in C\left([1, T], \mathbb{R}^{+}\right)$and a continuous nondecreasing function $\psi: \mathbb{R}^{+} \longrightarrow \mathbb{R}^{+}$such that

$$
|f(t, u)| \leq p(t) \psi(\|u\|) \quad \text { for each }(t, u) \in[1, T] \times \mathbb{R} .
$$

$\left(H_{5}\right)$. There exists a constant $K>0$ such that

$$
\frac{K}{\Omega\|p\| \psi(K)}>1
$$

3.1. Existence and Uniqueness Result via Banach's Fixed Point Theorem. We prove an existence and uniqueness result based on Banach's contraction mapping principle.

Theorem 10. Assume that $\left(H_{1}\right)$ holds. Then, boundary value problem (5) has a unique solution on $[1, T]$, provided that $l$ $\Omega<1$, where $\Omega$ is defined by (20).

Proof. We will use Banach's fixed point theorem to prove that $\mathscr{F}$, defined by (19), has a unique fixed point. Fixing $N$ $=\max _{t \in[1, T]}|f(t, 0)|<\infty$ and using hypothesis $\left(H_{1}\right)$, we obtain

$$
\begin{aligned}
|f(t, x(t))| & \leq|f(t, x(t))-f(t, 0)|+|f(t, 0)| \\
& \leq l|x(t)|+|f(t, 0)| \leq l\|x\|+N .
\end{aligned}
$$

Choose

$$
r \geq \frac{N \Omega}{1-l \Omega}
$$

We divide the proof into two steps. 
Step I. We show that $\mathscr{F}\left(B_{r}\right) \subset B_{r}$, where $B_{r}=\{x \in X: \|$ $x \|<r\}$. Let $x \in B_{r}$. Then, we have

$$
\begin{aligned}
|(\mathscr{F} x)(t)| \leq & \frac{1}{\Gamma(\alpha)} \int_{1}^{t}\left(\log \frac{t}{\tau}\right)^{\alpha-1} \frac{|f(\tau, x(\tau))|}{\tau} d \tau \\
& +\frac{(\log t)^{\gamma-1}}{|\Lambda|}\left\{\frac{|\lambda|}{\Gamma(\alpha+\delta)} \int_{1}^{\eta}\left(\log \frac{\eta}{\tau}\right)^{\alpha+\delta-1} \frac{|f(\tau, x(\tau))|}{\tau} d \tau\right. \\
& \left.+\sum_{i=1}^{m}\left|\theta_{i}\right| \frac{1}{\Gamma(\alpha)} \int_{1}^{\xi_{i}}\left(\log \frac{\xi_{i}}{\tau}\right)^{\alpha-1} \frac{|f(\tau, x(\tau))|}{\tau} d \tau\right\} \\
\leq & \frac{(\log T)^{\alpha}}{\Gamma(\alpha+1)}(l|| x \|+N)+\frac{(\log T)^{\gamma-1}}{|\Lambda|}\left\{\frac{|\lambda|(\log \eta)^{\alpha+\delta}}{\Gamma(\alpha+\delta+1)}\right. \\
& \left.+\sum_{i=1}^{m}\left|\theta_{i}\right| \frac{\left(\log \xi_{i}\right)^{\alpha}}{\Gamma(\alpha+1)}\right\}(l\|x\|+N) \\
= & {\left[\frac{(\log T)^{\alpha}}{\Gamma(\alpha+1)}+\frac{(\log T)^{\gamma-1}}{|\Lambda|}\left\{\frac{|\lambda|(\log \eta)^{\alpha+\delta}}{\Gamma(\alpha+\delta+1)}\right.\right.} \\
& \left.\left.+\sum_{i=1}^{m}\left|\theta_{i}\right| \frac{\left(\log \xi_{i}\right)^{\alpha}}{\Gamma(\alpha+1)}\right\}\right](l\|x\|+N) \\
\leq & {\left[\frac{(\log T)^{\alpha}}{\Gamma(\alpha+1)}+\frac{(\log T)^{\gamma-1}}{|\Lambda|}\left\{\frac{|\lambda|(\log \eta)^{\alpha+\delta}}{\Gamma(\alpha+\delta+1)}\right.\right.} \\
& \left.\left.+\sum_{i=1}^{m}\left|\theta_{i}\right| \frac{\left(\log \xi_{i}\right)^{\alpha}}{\Gamma(\alpha+1)}\right\}\right](l r+N)=\Omega(l r+N) \leq r .
\end{aligned}
$$

Thus,

$$
\|(\mathscr{F} x)\|=\max _{t \in[1, T]}|(\mathscr{F} x)(t)| \leq r,
$$

which means that $\left(\mathscr{F} B_{r}\right) \subset B_{r}$.

Step II. To show that the operator $\mathscr{F}$ is a contraction, let $x_{1}, x_{2} \in X$. Then, for any $t \in[1, T]$, we have

$$
\begin{aligned}
& \left|\left(\mathscr{F} x_{2}\right)(t)-\left(\mathscr{F} x_{1}\right)(t)\right| \leq \frac{1}{\Gamma(\alpha)} \int_{1}^{t}\left(\log \frac{t}{\tau}\right)^{\alpha-1} \frac{\left|f\left(\tau, x_{2}(\tau)\right)-f\left(s, x_{1}(\tau)\right)\right|}{\tau} d \tau \\
& +\frac{(\log t)^{\gamma-1}}{|\Lambda|}\left\{\frac{|\lambda|}{\Gamma(\alpha+\delta)} \int_{1}^{\eta} \otimes\left(\log \frac{\eta}{\tau}\right)^{\alpha+\delta-1} \frac{\left|f\left(s, x_{2}(\tau)\right)-f\left(s, x_{1}(\tau)\right)\right|}{\tau} d \tau\right. \\
& \left.+\sum_{i=1}^{m}\left|\theta_{i}\right| \frac{1}{\Gamma(\alpha)} \int_{1}^{\xi_{i}}\left(\log \frac{\xi_{i}}{\tau}\right)^{\alpha-1} \frac{\left|f\left(\tau, x_{2}(\tau)\right)-f\left(\tau, x_{1}(\tau)\right)\right|}{\tau} d \tau\right\} \\
& \leq l\left\|x_{2}-x_{1}\right\|\left[\frac{(\log T)^{\alpha}}{\Gamma(\alpha+1)}+\frac{(\log T)^{\gamma-1}}{|\Lambda|}\left\{\frac{|\lambda|(\log \eta)^{\alpha+\delta}}{\Gamma(\alpha+\delta+1)}+\sum_{i=1}^{m}\left|\theta_{i}\right| \frac{\left(\log \xi_{i}\right)^{\alpha}}{\Gamma(\alpha+1)}\right\}\right] .
\end{aligned}
$$

Thus,

$$
\left\|\left(\mathscr{F} x_{2}\right)-\left(\mathscr{F} x_{1}\right)\right\|=\max _{t \in[1, T]}\left|\left(\mathscr{F} x_{2}\right)(t)-\left(\mathscr{F} x_{1}\right)(t)\right| \leq l \Omega\left\|x_{2}-x_{1}\right\|,
$$

which, in view of $l \Omega<1$, shows that the operator $\mathscr{F}$ is a contraction. By Theorem 5, we get that the operator $\mathscr{F}$ has a unique fixed point. Therefore, the problem (5) has a unique solution on $[1, T]$. The proof is completed. $\square$

3.2. Existence Result via Krasnoselskii's Fixed Point Theorem. In this subsection, we prove an existence result based on Krasnoselskii's fixed point theorem.

Theorem 11. Assume that $\left(H_{1}\right)-\left(H_{2}\right)$ hold. Then, the problem (5) has at least one solution on $[1, T]$, provided that

$$
\frac{(\log T)^{\gamma-1}}{|\Lambda|}\left\{\frac{|\lambda|(\log \eta)^{\alpha+\delta}}{\Gamma(\alpha+\delta+1)}+\sum_{i=1}^{m}\left|\theta_{i}\right| \frac{\left(\log \xi_{i}\right)^{\alpha}}{\Gamma(\alpha+1)}\right\} l<1 .
$$

Proof. By assumption $\left(H_{2}\right)$, we can fix $\rho \geq \Omega\|\phi\|$, where $\| \phi$ $\|=\sup _{t \in[1, T]}|\phi(t)|$ and consider $B_{\rho}=\{x \in C([1, T], \mathbb{R}):\|x\|$ $\leq \rho\}$. We split the operator $\mathscr{F}: C([1, T], \mathbb{R}) \longrightarrow C([1, T]$, $\mathbb{R})$ defined by $(19)$ as $\mathscr{F}=\mathscr{F}_{1}+\mathscr{F}_{2}$, where $\mathscr{F}_{1}$ and $\mathscr{F}_{2}$ are given by

$$
\begin{gathered}
\left(\mathscr{F}_{1} x\right)(t)=\frac{1}{\Gamma(\alpha)} \int_{1}^{t}\left(\log \frac{t}{\tau}\right)^{\alpha-1} \frac{f(\tau, x(\tau))}{\tau} d \tau \\
\left(\mathscr{F}_{2} x\right)(t)=\frac{(\log t)^{\gamma-1}}{\Lambda}\left\{\frac{\lambda}{\Gamma(\alpha+\delta)} \int_{1}^{\eta}\left(\log \frac{\eta}{\tau}\right)^{\alpha+\delta-1} \frac{f(\tau, x(\tau))}{\tau} d \tau\right. \\
\left.\quad-\sum_{i=1}^{m} \theta_{i} \frac{1}{\Gamma(\alpha)} \int_{1}^{\xi_{i}}\left(\log \frac{\xi_{i}}{\tau}\right)^{\alpha-1} \frac{f(\tau, x(\tau))}{\tau} d \tau\right\}
\end{gathered}
$$

For any $x, y \in B_{\rho}$, we have

$$
\begin{aligned}
& \left|\left(\mathscr{F}_{1} x\right)(t)+\left(\mathscr{F}_{2} y\right)(t)\right| \leq \frac{1}{\Gamma(\alpha)} \int_{1}^{t}\left(\log \frac{t}{\tau}\right)^{\alpha-1} \frac{|f(\tau, x(\tau))|}{\tau} d \tau \\
& +\frac{(\log t)^{\gamma-1}}{|\Lambda|}\left\{\frac{|\lambda|}{\Gamma(\alpha+\delta)} \int_{1}^{\eta}\left(\log \frac{\eta}{\tau}\right)^{\alpha+\delta-1} \frac{|f(\tau, y(\tau))|}{\tau} d \tau\right. \\
& \left.+\sum_{i=1}^{m}\left|\theta_{i}\right| \frac{1}{\Gamma(\alpha)} \int_{1}^{\xi_{i}}\left(\log \frac{\xi_{i}}{\tau}\right)^{\alpha-1} \frac{|f(\tau, y(\tau))|}{\tau} d \tau\right\} \\
& \quad \leq\left[\frac{(\log T)^{\alpha}}{\Gamma(\alpha+1)}+\frac{(\log T)^{\gamma-1}}{|\Lambda|}\left\{\frac{|\lambda|(\log \eta)^{\alpha+\delta}}{\Gamma(\alpha+\delta+1)}+\sum_{i=1}^{m}\left|\theta_{i}\right| \frac{\left(\log \xi_{i}\right)^{\alpha}}{\Gamma(\alpha+1)}\right\}\right]\|\phi\| \\
& \quad=\Omega\|\phi\| \leq r .
\end{aligned}
$$

Hence, $\left\|\left(\mathscr{F}_{1} x\right)+\left(\mathscr{F}_{2} y\right)\right\| \leq \rho$, which shows that $\left(\mathscr{F}_{1} x\right.$ )$+\left(\mathscr{F}_{2} y\right) \in B_{\rho}$. It is easy to prove, using conditions $\left(H_{1}\right.$ ) and (32), that the operator $\mathscr{F}_{2}$ is a contraction mapping.

Next, the operator $\mathscr{F}_{1}$ is continuous by the continuity of $f$. Also, $\mathscr{F}_{1}$ is uniformly bounded on $B_{\rho}$, since

$$
\left\|\mathscr{F}_{1} x\right\| \leq \frac{(\log T)^{\alpha}}{\Gamma(\alpha+1)}\|\phi\| .
$$


Finally, we prove the compactness of the operator $\mathscr{F}_{1}$. For $t_{1}, t_{2} \in[1, T], t_{1}<t_{2}$, we have

$$
\begin{aligned}
\left|\left(\mathscr{F}_{1} x\right)\left(t_{2}\right)-\left(\mathscr{F}_{1} x\right)\left(t_{1}\right)\right| & \\
\leq & \frac{1}{\Gamma(\alpha)} \int_{1}^{t_{1}}\left[\left(\log \frac{t_{2}}{\tau}\right)^{\alpha-1}-\left(\log \frac{t_{1}}{\tau}\right)^{\alpha-1}\right] \frac{|f(\tau, x(\tau))|}{\tau} d \tau \\
& \quad+\frac{1}{\Gamma(\alpha)} \int_{t_{1}}^{t_{2}}\left(\log \frac{t_{2}}{\tau}\right)^{\alpha-1} \frac{|f(\tau, x(\tau))|}{\tau} d \tau \\
\leq & \frac{\|\phi\|}{\Gamma(\alpha+1)}\left[2\left(\log t_{2}-\log t_{1}\right)^{\alpha}+\left|\left(\log t_{2}\right)^{\alpha}-\left(\log t_{1}\right)^{\alpha}\right|\right]
\end{aligned}
$$

which tends to zero, independently of $x \in B_{\rho}$, as $t_{1} \longrightarrow t_{2}$. Thus, $\mathscr{F}_{1}$ is equicontinuous. From the Arzelá-Ascoli theorem, we conclude that the operator $\mathscr{F}_{1}$ is compact on $B_{\rho}$. Thus, the hypotheses of Krasnoselskii fixed point theorem are satisfied, and therefore, there exists at least one solution on $[1, T]$. The proof is finished. $\square$

3.3. Existence Result via Schaefer's Fixed Point Theorem. Our second existence result is based on Schaefer's fixed point theorem.

Theorem 12. Assume that $\left(\mathrm{H}_{3}\right)$ holds. Then, the boundary value problem (5) has at least one solution on $[1, T]$.

Proof. We will prove that the operator $\mathscr{F}$, defined by (19), has a fixed point, by using Schaefer's fixed point theorem. We divide the proof into two steps.

Step I. We show that the operator $\mathscr{F}: X \longrightarrow X$ is completely continuous.

We show first that $\mathscr{F}$ is continuous. Let $\left\{x_{n}\right\}$ be a sequence such that $x_{n} \longrightarrow x$ in $X$. Then, for each $t \in[1, T]$, we have

$$
\begin{aligned}
& \left|\left(\mathscr{F} x_{n}\right)(t)-(\mathscr{F} x)(t)\right| \\
& \leq \frac{1}{\Gamma(\alpha)} \int_{1}^{t}\left(\log \frac{t}{\tau}\right)^{\alpha-1} \frac{\left|f\left(\tau, x_{n}(\tau)\right)-f(\tau, x(\tau))\right|}{\tau} d \tau \\
& \quad+\frac{(\log t)^{\gamma-1}}{|\Lambda|}\left\{\frac{|\lambda|}{\Gamma(\alpha+\delta)} \int_{1}^{\eta}\left(\log \frac{\eta}{\tau}\right)^{\alpha+\delta-1} \frac{\left|f\left(\tau, x_{n}(\tau)\right)-f(\tau, x(\tau))\right|}{\tau} d \tau\right. \\
& \left.\quad+\sum_{i=1}^{m}\left|\theta_{i}\right| \frac{1}{\Gamma(\alpha)} \int_{1}^{\xi_{i}}\left(\log \frac{\xi_{i}}{\tau}\right)^{\alpha-1} \frac{\left|f\left(\tau, x_{n}(\tau)\right)-f(\tau, x(\tau))\right|}{\tau} d \tau\right\} .
\end{aligned}
$$

Since $f$ is continuous, we get

$$
\left|f\left(s, x_{n}(s)\right)-f(s, x(s))\right| \longrightarrow 0 \quad \text { as } x_{n} \longrightarrow x \text {. }
$$

Then

$$
\left\|\left(\mathscr{F} x_{n}\right)-(\mathscr{F} x)\right\| \longrightarrow 0 \text { as } x_{n} \longrightarrow x
$$

Hence, $\mathscr{F}$ is continuous.

Secondly, we show that the operator $\mathscr{F}$ maps bounded sets into bounded sets in $X$. For a positive number $R$, let $B_{R}=\{x \in X:\|x\| \leq R\}$ be a bounded ball in $X$. Then, for $t$ $\in[1, T]$, we have

$$
\begin{aligned}
|(\mathscr{F} x)(t)| \leq & \frac{1}{\Gamma(\alpha)} \int_{1}^{t}\left(\log \frac{t}{\tau}\right)^{\alpha-1} \frac{|f(\tau, x(\tau))|}{\tau} d \tau \\
& +\frac{(\log t)^{\gamma-1}}{|\Lambda|}\left\{\frac{|\lambda|}{\Gamma(\alpha+\delta)} \int_{1}^{\eta}\left(\log \frac{\eta}{\tau}\right)^{\alpha+\delta-1} \frac{|f(\tau, x(\tau))|}{\tau} d \tau\right. \\
& \left.+\sum_{i=1}^{m}\left|\theta_{i}\right| \frac{1}{\Gamma(\alpha)} \int_{1}^{\xi_{i}}\left(\log \frac{\xi_{i}}{\tau}\right)^{\alpha-1} \frac{|f(\tau, x(\tau))|}{\tau} d \tau\right\} \\
\leq & \frac{(\log T)^{\alpha}}{\Gamma(\alpha+1)} M+\frac{(\log T)^{\gamma-1}}{|\Lambda|}\left\{\frac{|\lambda|(\log \eta)^{\alpha+\delta}}{\Gamma(\alpha+\delta+1)}\right. \\
& \left.+\sum_{i=1}^{m}\left|\theta_{i}\right| \frac{\left(\log \xi_{i}\right)^{\alpha}}{\Gamma(\alpha+1)}\right\} M,
\end{aligned}
$$

and

$\|\mathscr{F} x\| \leq \frac{(\log T)^{\alpha}}{\Gamma(\alpha+1)} M+\frac{(\log T)^{\gamma-1}}{|\Lambda|}\left\{\frac{|\lambda|(\log \eta)^{\alpha+\delta}}{\Gamma(\alpha+\delta+1)}+\sum_{i=1}^{m}\left|\theta_{i}\right| \frac{\left(\log \xi_{i}\right)^{\alpha}}{\Gamma(\alpha+1)}\right\} M$.

Thirdly, we show that $\mathscr{F}$ maps bounded sets into equicontinuous sets. Let $t_{1}, t_{2} \in[1, T]$ with $t_{1}<t_{2}$ and $u \in B_{R}$. Then, we have

$$
\begin{aligned}
\left|(\mathscr{F} x)\left(t_{2}\right)-(\mathscr{F} x)\left(t_{1}\right)\right| \leq \frac{1}{\Gamma(\alpha)} \int_{1}^{t_{1}}\left[\left(\log \frac{t_{2}}{\tau}\right)^{\alpha-1}\right. \\
\left.-\left(\log \frac{t_{1}}{\tau}\right)^{\alpha-1}\right] \frac{|f(\tau, x(\tau))|}{\tau} d \tau \\
+\frac{1}{\Gamma(\alpha)} \int_{t_{1}}^{t_{2}}\left(\log \frac{t_{2}}{\tau}\right)^{\alpha-1} \frac{|f(\tau, x(\tau))|}{\tau} d \tau \\
+\frac{\left|\left(\log t_{2}\right)^{\gamma-1}-\left(\log t_{1}\right)^{\gamma-1}\right|}{|\Lambda|}\left\{\frac{|\lambda|}{\Gamma(\alpha+\delta)} \int_{1}^{\eta}\left(\log \frac{\eta}{\tau}\right)^{\alpha+\delta-1} \frac{|f(\tau, x(\tau))|}{\tau} d \tau\right. \\
\left.+\sum_{i=1}^{m}\left|\theta_{i}\right| \frac{1}{\Gamma(\alpha)} \int_{1}^{\xi_{i}}\left(\log \frac{\xi_{i}}{\tau}\right)^{\alpha-1} \frac{|f(\tau, x(\tau))|}{\tau} d \tau\right\} \\
\quad \leq \frac{M}{\Gamma(\alpha+1)}\left[2\left(\log t_{2}-\log t_{1}\right)^{\alpha}+\left|\left(\log t_{2}\right)^{\alpha}-\left(\log t_{1}\right)^{\alpha}\right|\right] \\
+\frac{\left|\left(\log t_{2}\right)^{\gamma-1}-\left(\log t_{1}\right)^{\gamma-1}\right|}{|\Lambda|}\left\{\frac{|\lambda|(\log \eta)^{\alpha+\delta}}{\Gamma(\alpha+\delta+1)}+\sum_{i=1}^{m}\left|\theta_{i}\right| \frac{\left(\log \xi_{i}\right)^{\alpha}}{\Gamma(\alpha+1)}\right\} M,
\end{aligned}
$$

which tends to zero, independently of $x \in B_{R}$, as $t_{1} \longrightarrow t_{2}$. 
Thus, the Arzelá-Ascoli theorem applies and hence, $\mathscr{F}: X$ $\longrightarrow X$ is completely continuous.

Step II. We show that the set $\mathscr{E}=\{x \in X \mid x=v(\mathscr{F} x), 0$ $\leq v \leq 1\}$ is bounded. Let $x \in \mathscr{E}$, then $x=v(\mathscr{F} x)$. For any $t$ $\epsilon[1, T]$, we have $x(t)=v(\mathscr{F} x)(t)$. Then, in view of the hypothesis $\left(H_{3}\right)$, as in Step I, we obtain

$$
|x(t)| \leq \frac{(\log T)^{\alpha}}{\Gamma(\alpha+1)} M+\frac{(\log T)^{\gamma-1}}{|\Lambda|}\left\{\frac{|\lambda|(\log \eta)^{\alpha+\delta}}{\Gamma(\alpha+\delta+1)}+\sum_{i=1}^{m}\left|\theta_{i}\right| \frac{\left(\log \xi_{i}\right)^{\alpha}}{\Gamma(\alpha+1)}\right\} M .
$$

Thus,

$$
\|x\| \leq \frac{(\log T)^{\alpha}}{\Gamma(\alpha+1)} M+\frac{(\log T)^{\gamma-1}}{|\Lambda|}\left\{\frac{|\lambda|(\log \eta)^{\alpha+\delta}}{\Gamma(\alpha+\delta+1)}+\sum_{i=1}^{m}\left|\theta_{i}\right| \frac{\left(\log \xi_{i}\right)^{\alpha}}{\Gamma(\alpha+1)}\right\} M,
$$

which shows that the set $\mathscr{E}$ is bounded. By Theorem 7, we get that the operator $\mathscr{F}$ has at least one fixed point. Therefore, the boundary value problem (5) has at least one solution on $[1, T]$. This completes the proof. $\square$

3.4. Existence Result via Leray-Schauder Nonlinear Alternative. Our final existence result is proved via LeraySchauder nonlinear alternative.

Theorem 13. Assume that $\left(H_{4}\right)$ and $\left(H_{5}\right)$ hold. Then, the boundary value problem (5) has at least one solution on $[1, T]$.

Proof. As in Theorem 12, we can prove that the operator $\mathscr{F}$ is completely continuous. We will prove that there exists an open set $U \subseteq C([1, T], \mathbb{R})$ with $x \neq \mu(\mathscr{F} x)$ for $\mu \in(0,1)$ and $x \in \partial U$.
Let $x \in C([1, T], \mathbb{R})$ be such that $x=\mu(\mathscr{F} x)$ for some 0 $<\mu<1$. Then, for each $t \in[1, T]$, we have

$$
\begin{aligned}
|x(t)| \leq & \frac{1}{\Gamma(\alpha)} \int_{1}^{t}\left(\log \frac{t}{\tau}\right)^{\alpha-1} \frac{|f(\tau, x(\tau))|}{\tau} d \tau \\
& +\frac{(\log t)^{\gamma-1}}{|\Lambda|}\left\{\frac{|\lambda|}{\Gamma(\alpha+\delta)} \int_{1}^{\eta}\left(\log \frac{\eta}{\tau}\right)^{\alpha+\delta-1} \frac{|f(\tau, x(\tau))|}{\tau} d \tau\right. \\
& \left.+\sum_{i=1}^{m}\left|\theta_{i}\right| \frac{1}{\Gamma(\alpha)} \int_{1}^{\xi_{i}}\left(\log \frac{\xi_{i}}{\tau}\right)^{\alpha-1} \frac{|f(\tau, x(\tau))|}{\tau} d \tau\right\} \\
\leq & {\left[\frac{(\log T)^{\alpha}}{\Gamma(\alpha+1)}+\frac{(\log T)^{\gamma-1}}{|\Lambda|}\left\{\frac{|\lambda|(\log \eta)^{\alpha+\delta}}{\Gamma(\alpha+\delta+1)}\right.\right.} \\
& \left.\left.+\sum_{i=1}^{m}\left|\theta_{i}\right| \frac{\left(\log \xi_{i}\right)^{\alpha}}{\Gamma(\alpha+1)}\right\}\right]\|p\| \psi(\|x\|) .
\end{aligned}
$$

Consequently,

$$
U=\{x \in C([1, T], \mathbb{R}):\|x\|<K\} .
$$

In view of $\left(H_{5}\right)$, there is no solution $x$ such that $\|x\| \neq K$. Let us set

$$
U=\{x \in C([1, T], \mathbb{R}):\|x\|<K\} .
$$

The operator $\mathscr{F}: \bar{U} \longrightarrow C([1, T], \mathbb{R})$ is continuous and completely continuous. From the choice of $U$, there is no $u$ $\epsilon \partial U$ such that $x=\mu(\mathscr{F} x)$ for some $\mu \in(0,1)$. Consequently, by the nonlinear alternative of Leray-Schauder type (Theorem 8 ), we deduce that $\mathscr{F}$ has a fixed point $x \in \bar{U}$ which is a solution of the boundary value problem (5). The proof is completed. $\square$

\section{Examples}

Example 1. Consider the Hilfer-Hadamard nonlocal integromultipoint fractional boundary value problem of the form

$$
\left\{\begin{array}{l}
{ }^{\mathrm{HH}} D_{1}^{3 / 2,1 / 2} x(t)=f(t, x(t)), \quad t \in[1,4.5], \\
x(1)=0, \quad \frac{6^{H}}{29} I_{1}^{5 / 2} x\left(\frac{5}{2}\right)=\frac{1}{11} x\left(\frac{3}{2}\right)+\frac{2}{13} x(2)+\frac{3}{17} x(3)+\frac{4}{19} x\left(\frac{7}{2}\right)+\frac{5}{23} x(4) .
\end{array}\right.
$$

Set constants $\alpha=3 / 2, \beta=1 / 2, T=9 / 2, m=5, \theta_{1}=1 / 11, \theta_{2}$ $=2 / 13, \theta_{3}=3 / 17, \theta_{4}=4 / 19, \theta_{5}=5 / 23, \xi_{1}=3 / 2, \xi_{2}=2, \xi_{3}=$ $3, \xi_{4}=7 / 2, \xi_{5}=4, \lambda=6 / 29, \delta=5 / 2$, and $\eta=5 / 2$. Then, from these data, we compute that $\gamma=7 / 4,|\Lambda| \approx 1.152315230$, and $\Omega \approx 2.250815481$,

(i) Let a nonlinear unbounded function $f:[1,4.5] \times \mathbb{R}$ $\longrightarrow \mathbb{R}$ be defined by

$$
f(t, x)=\frac{e^{1-t}}{t+4}\left(\frac{x^{2}+2|x|}{1+|x|}\right)+\frac{1}{2} t^{2}+1
$$

Now, we can verify that function $f(t, x)$ satisfies the Lipchitz condition $\left(H_{1}\right)$ with $l=2 / 5$, that is, $|f(t, x)-f(t, y)|$ $\leq(2 / 5)|x-y|$, for all $t \in[1,4.5]$ and $x, y \in \mathbb{R}$. Then, we obtain $l \Omega \approx 0.9003261924<1$. The conclusion can be gotten 
from Theorem 10 that the problem (47), with $f$ given by (48), has a unique solution on $[1,4.5]$.

(ii) If the term $x^{2}$ in (48) is replaced by $|x|$, that is,

$$
f(t, x)=\frac{3 e^{1-t}}{t+4}\left(\frac{|x|}{1+|x|}\right)+\frac{1}{2} t^{2}+1
$$

then the nonlinear function $f$ is bounded by a function of $t$ by

$$
|f(t, x)| \leq \frac{3 e^{1-t}}{t+4}+\frac{1}{2} t^{2}+1:=\phi(t)
$$

which is satisfied a condition $\left(\mathrm{H}_{2}\right)$. It is easy to check that function $f$ in (49) is fulfilled the Lipchitz condition with constant $l=3 / 5$. Since

$$
\frac{(\log T)^{\gamma-1}}{|\Lambda|}\left\{\frac{|\lambda|(\log \eta)^{\alpha+\delta}}{\Gamma(\alpha+\delta+1)}+\sum_{i=1}^{m}\left|\theta_{i}\right| \frac{\left(\log \xi_{i}\right)^{\alpha}}{\Gamma(\alpha+1)}\right\} l \approx 0.5179201139<1
$$

we can deduce that the Hilfer-Hardamard nonlocal integromultipoint fractional boundary value problem (47), with $f$ given by (49), has at least one solution on $[1,4.5]$. In addition, we observe that Theorem 10 cannot be used in this case because $l \Omega \approx 1.350489289>1$

(iii) If $f$ is a non-Lipschitzian function as

$$
f(t, x)=\frac{e^{1-t}}{t+4} \sin ^{12}\left(\frac{x^{2}+2|x|}{1+|x|}\right)+\frac{1}{2} t^{2}+1
$$

to claim the existence of boundary value problem (47), with $f$ given by (52), we can find that

$$
|f(t, x)| \leq \frac{1}{5}+\frac{1}{2}\left(\frac{9}{2}\right)^{2}+1=\frac{453}{40}
$$

Hence, $\left(\mathrm{H}_{3}\right)$ is satisfied with $M=453 / 40$. Therefore, by the benefit of Theorem 12, problem (47) with $f$ given by (52) has at least one solution on $[1,4.5]$.

(iv) If $f$ is a non-Lipschitzian function defined by

$$
f(t, x)=\frac{e^{1-t}}{t+4}\left(\frac{x^{26}}{1+x^{24}}+\frac{1}{9} t+\frac{1}{2}\right)
$$

we see that $\left(H_{4}\right)$ holds as

$$
|f(t, x)| \leq \frac{e^{1-t}}{t+4}\left(x^{2}+1\right)
$$

by setting $p(t)=\left(e^{1-t}\right) /(t+4)$ and $\psi(x)=x^{2}+1$. Then, we obtain $\|p\|=1 / 5$ and there exists a constant $K \in($
$0.6273106970,1.594106403)$ satisfying condition $\left(H_{5}\right)$. Thus, using Theorem 13, the boundary value problem (47), with $f$ is given in (54), has at least one solution on $[1,4.5]$

\section{Conclusion}

In this paper, we have presented the existence and uniqueness criteria for solutions for Hilfer-Hadamard fractional differential equation complemented with nonlocal integromultipoint boundary conditions. Firstly, we convert the given nonlinear problem into a fixed point problem. Once the fixed point operator is available, we make use of Banach contraction mapping principle to obtain the uniqueness result, while the existence results are established by applying Schaefer and Krasnoselskii fixed point theorems as well as Leray-Schauder nonlinear alternative. Our results are new in the given configuration and enrich the literature on boundary value problems involving Hilfer-Hadamard fractional differential equations.

\section{Data Availability}

No data were used to support this study.

\section{Conflicts of Interest}

The authors declare that there are no conflicts of interest regarding the publication of this paper.

\section{Acknowledgments}

This research was funded by King Mongkut's University of Technology North Bangkok, Contract no. KMUTNB-61GOV-B-29.

\section{References}

[1] K. Diethelm, The Analysis of Fractional Differential Equations, Lecture Notes in Mathematics, Springer, New York, 2010.

[2] A. A. Kilbas, H. M. Srivastava, and J. J. Trujillo, Theory and Applications of the Fractional Differential Equations, vol. 204, North-Holland Mathematics Studies, 2006.

[3] V. Lakshmikantham, S. Leela, and J. V. Devi, Theory of Fractional Dynamic Systems, Cambridge Scientific Publishers, 2009.

[4] K. S. Miller and B. Ross, An Introduction to the Fractional Calculus and Differential Equations, John Wiley, New York, 1993.

[5] I. Podlubny, Fractional Differential Equations, Academic Press, New York, 1999.

[6] B. Ahmad, A. Alsaedi, S. K. Ntouyas, and J. Tariboon, Hadamard-Type Fractional Differential Equations, Inclusions and Inequalities, Springer, Cham, Switzerland, 2017.

[7] Y. Zhou, Basic Theory of Fractional Differential Equations, World Scientific, Singapore, 2014.

[8] S. G. Samko, A. A. Kilbas, and O. I. Marichev, Fractional Integrals and Derivatives, Gordon and Breach Science, Yverdon, 1993.

[9] R. Hilfer, Ed., Applications of Fractional Calculus in Physics, World Scientific, Singapore, 2000. 
[10] R. Hilfer, "Experimental evidence for fractional time evolution in glass forming materials," The Journal of Chemical Physics, vol. 284, pp. 399-408, 2002.

[11] R. Hilfer, Y. Luchko, and Z. Tomovski, “Operational method for the solution of fractional differential equations with generalized Riemann-Liouvill fractional derivatives," Fractional Calculus and Applied Analysis, vol. 12, pp. 299-318, 2009.

[12] K. M. Furati, N. D. Kassim, and N. E. Tatar, "Existence and uniqueness for a problem involving Hilfer fractional derivative," Computers \& Mathematcs with Applications, vol. 64, no. 6, pp. 1616-1626, 2012.

[13] H. Gu and J. J. Trujillo, "Existence of mild solution for evolution equation with Hilfer fractional derivative," Applied Mathematics and Computation, vol. 257, pp. 344-354, 2015.

[14] J. Wang and Y. Zhang, "Nonlocal initial value problems for differential equations with Hilfer fractional derivative," Applied Mathematics and Computation, vol. 266, pp. 850859, 2015.

[15] V. C. Sousa and E. C. de Oliveira, "On the $\psi$-Hilfer fractional derivative," Communications in Nonlinear Science and Numerical Simulation, vol. 60, pp. 72-91, 2018.

[16] S. Asawasamrit, A. Kijjathanakorn, S. K. Ntouyas, and J. Tariboon, "Nonlocal boundary value problems for Hilfer fractional differential equations," Bulletin of the Korean Mathematical Society, vol. 55, no. 6, pp. 1639-1657, 2018.

[17] A. Wongcharoen, B. Ahmad, S. K. Ntouyas, and J. Tariboon, "Three-point boundary value problems for the Langevin equation with the Hilfer fractional derivative," Advances in Mathematical Physic, vol. 2020, article 9606428, pp. 1-11, 2020.

[18] W. Saengthong, E. Thailert, and S. K. Ntouyas, "Existence and uniqueness of solutions for system of Hilfer-Hadamard sequential fractional differential equations with two point boundary conditions," Advances in Difference Equations, vol. 2019, no. 1, Article ID 525, 2019.

[19] M. A. Krasnoselskii, "Two remarks on the method of successive approximations," Uspekhi Matematicheskikh Nauk, vol. 10, pp. 123-127, 1955.

[20] A. Granas and J. Dugundji, Fixed Point Theory, Springer-Verlag, New York, 2003.

[21] K. Deimling, Nonlinear Functional Analysis, Springer-Verlag, New York, 1985.

[22] D. R. Smart, Fixed Poin Theory, Cambridge Univ Press, 1974. 